\title{
Design of Hyperbolic Curved Hull Plates Pressing Control System Based on CAN Bus
}

\author{
Jie Xiong ${ }^{1,2, *}$, Yong $\mathrm{Hu}^{1}$ and Zhanglong $\mathrm{Xia}^{1}$ \\ ${ }^{1}$ School of Transportation, Wuhan University of Technology, Wuhan, 430063, China \\ ${ }^{2}$ Institute of Mechanical and Electronic Engineering, Wuhan Institute of Technology, Wuhan, 430205, China
}

\begin{abstract}
The network structure and working process of the hyperbolic curved hull plates pressing control system which based on CAN bus was designed in this paper. The design scheme, hardware structure of the PC and the lower computer, the application flow of the lower computer and interface of the PC was also been introduced. Finally, experimental results proved that the control system has the characters of simple, stable and reliable, forming good quality, high production efficiency and so on.
\end{abstract}

Keywords: CAN bus, hull plates pressing machine, stepper motor, control system.

\section{INTRODUCTION}

Currently, hyperbolic curved hull plates (such as bow, stern sail-shaped plate, the saddle-shaped plate) forming must first take a one-way roller bend in the direction of greater curvature, and then use the line heating in another direction of smaller curvature. This hyperbolic degree hull plate processing method is commonly used in shipyards at home and abroad, but it has poor quality of shaping and low productivity. Hyperbolic degree hull plate platen machine is a multi-reconfigurable die forming technology, and the difference between it with other reconfigurable mold is that the degree of hyperbolic press machine uses a non-pressure and more pressure head stamping technology, and multi-heads is controlled by stepper motors via CAN bus. Compared with other reconfigurable mold, it has the characters of processing sheet metal without wrinkles phenomenon, smaller indentation and smoother surface [1].The surfaces forming principles of the hyperbolic press machine, which can form different surfaces by control the moveable press head under mold, is substantially the same with other reconfigurable mold [2]. The power of moveable pressure head comes from the step motor, the stepper motor changes electrical pulse signal rapidly and accurately into linear displacement and controls the mobile head to form different surfaces. Therefore, in order to improve both the processing of the hull plates hyperbolic and the production efficiency, this paper designs and implements hyperbolic degree hull plate press machine control system based on CAN bus.

\section{STRUCTURE OF CONTROL SYSTEM}

The hyperbolic curved hull plates pressing machine is mainly formed by presses, three-dimensional surface measurement devices and CAD/CAM software. The control sys- tem is the core of the entire platen machine equipment, and its main function is to calculate and generate the target shaped pieces forming data through the PC CAD/CAM software, and to send the command to the lower computer stepper motor controller. After that, it adjusts the height of the lower die to constitute the appropriate envelope; multihead forming presses are operating mechanism of repression sheet. Measuring device uses a three-dimensional laser measuring instrument, which could move with upper die to measure the shape of the mold of the actual pressing plate, and then upload the scanned measurement data to the PC. According to the geometric shape and the materials of the target plate, the $\mathrm{CAD} / \mathrm{CAM}$ software can calculate the data needed for multi-shaped.

Fig. (1) is a block diagram of the control system press machine, which includes a PC, a bus adapter card, some CAN bus transceivers, some stepper motor servo units and stepper motors. PC constitutes each node via bus adapter card and motor servo unit. The bus adapter card uses ADLINK PCI-7841 dual-port isolated CAN bus interface cards. The controllers and drivers constitute the servo motor units [3]. The tune-shaped data is first generated by CAD/CAM software on PC. After imported into control software, the data is converted to CAN communication signal by bus adapter [4]. CAN communication signal reaches the specified node via the bus, and finally data is analyzed and the stepper motor is driven to corresponding action by the controller.

There are 15 rows by 15 columns a total of 225 heads on the bottom basic mold, so the motor control system must control the 225 motors, but which generally field bus cannot reach. In order to reduce load and control cost, the system designed special controller and driver. By using one controller to control two drivers, one driver to drive two stepper motors, the system achieves that using 1 stepper motor controller to control 4 stepper motor, so the system just need 57 controller to meet all the needs of motor controlling. Mean- 


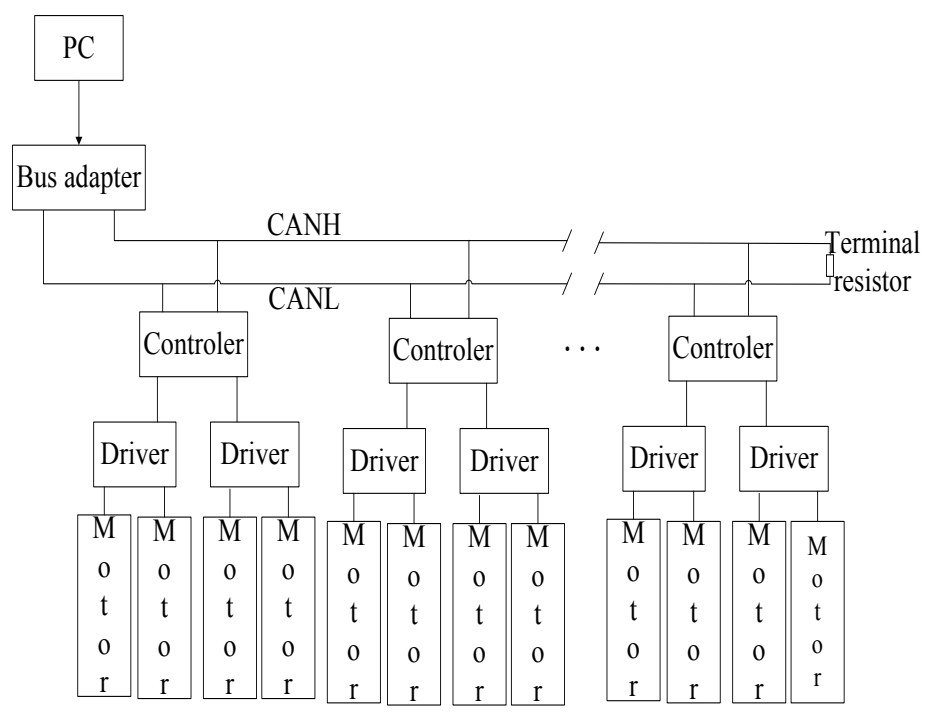

Fig. (1). The control system structure diagram.

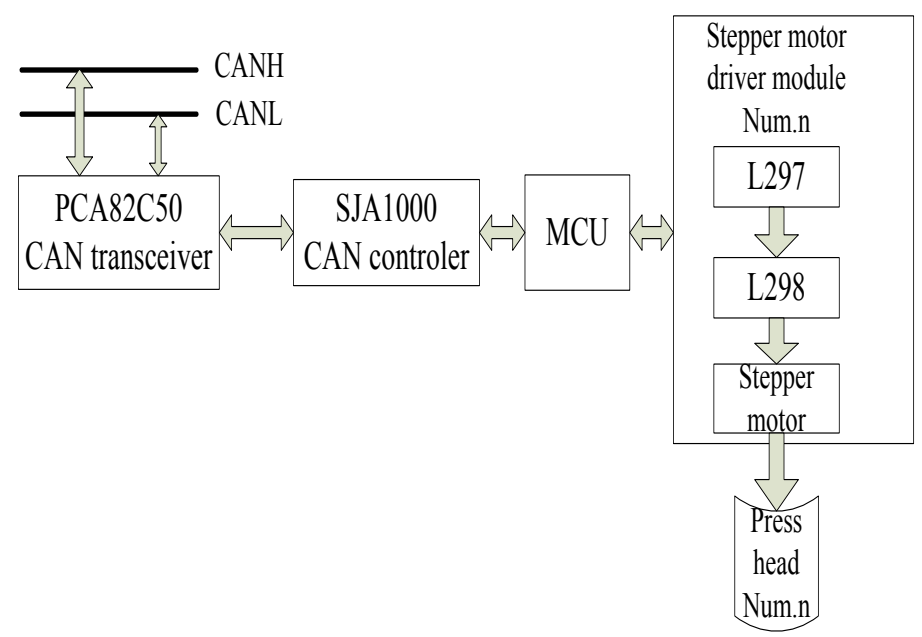

Fig. (2). The hardware structure diagram.

while, the design also meet the requirements of CAN bus load maximum node, which is at most 110 nodes [4].

\section{DESIGN OF THE LOWER COMPUTER NODE}

The lower computer's function is to receive control commands of the lower node sent by PC via CAN bus. The microprocessor analysis and process the data, then outputs corresponding pulse signal to the stepper motor driver based on the type and control content. Stepper motor driver drives stepping motor rotation to achieve displacement of the press head. The lower computer node structure diagram is shown in Fig. (2).

\subsection{CAN Controller}

The system CAN controller is made of by the master MCU, CAN controller, CAN transceiver and optical coupler components. Microprocessor manufactured using Atmel ATmega64, which is based on low-power enhanced AVR RISC structure $8 \mathrm{CMOS}$ microcontroller. It can up to 16 MIPS when operate on the $16 \mathrm{MHz}$ performance. It also has $64 \mathrm{~K}$ bytes of in-system programmable within flash, $2 \mathrm{~K}$ bytes of EEPROM, two8-bit timer/counter with the function of comparators and separate presale, and programmable serial USART [5]. It also has a separate on-chip oscillator with a programmable watchdog; all of these can well meet the system performance requirements of MCU.CAN controller uses Philips Semiconductor production SJA1000T, which in addition to support Basic-CAN mode, increased Peli-CAN mode and CAN2.0B protocol. The microprocessor interface of SJA1000T provided is typical Motorola or INTEL address data multiplexed bus mode, the main signal is a chip select signal CS, a read signal RD, write signal WR, address latch signal ALE, mode selection signal MODE and address Data signals $\mathrm{AD} 0 \sim \mathrm{AD} 7$. When $\mathrm{MODE}=0$, it works on 


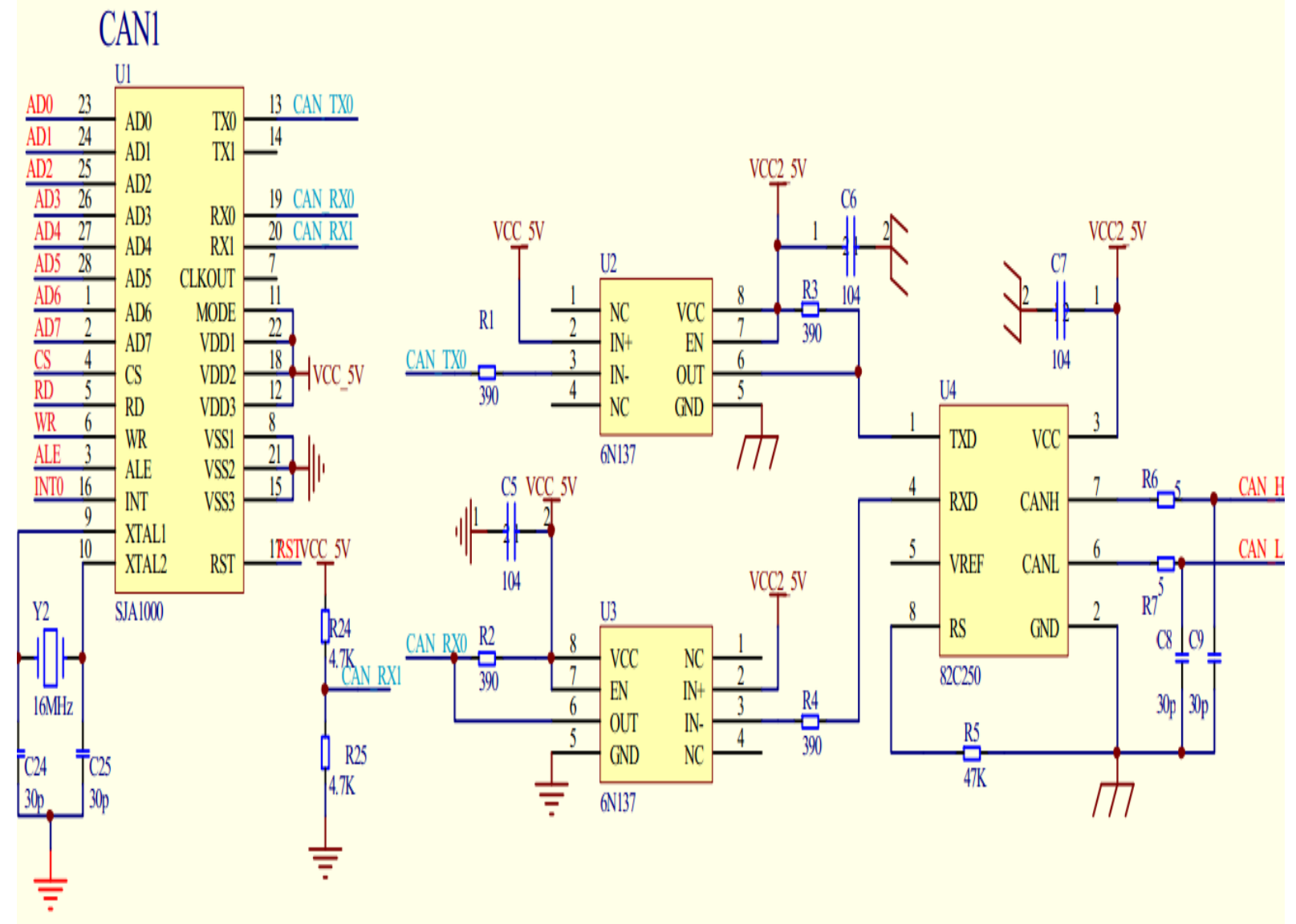

Fig. (3). CAN controller hardware principle diagram.

Motorola mode, when MODE $=1$, it works on INTEL mode [6]. The system selected the INTEL mode in this paper.

CAN bus transceiver uses PCA82C250T, which is the interface between the CAN protocol controller and the physical bus. The device provides differential transmit capability for the bus, and also provides differential receive capability for CAN controller.

The RX0 pin through and TX0 of SJA1000 was connected with the TXD and RXD of CAN bus transceiver $82 \mathrm{C} 250$ by the high-speed opt coupler chip $6 \mathrm{~N} 137$, which can increase the reliability of the nodes and achieve electrical isolation of each node on the CAN bus. At the same time, a capacitor between power and ground of $6 \mathrm{~N} 137$ can play the important role of isolation and immunity.

PC sends data to PCA82C250T via the CAN bus transceiver, and then PCA82C25T transfers the data to the CAN controller SJA1000T.By controlling the CS, a read signal $\mathrm{RD}$, read address data signal AD7 AD0, ATmega64 can receive data from SJA1000T.After parsing the data a, ATmega64 control the motor to perform corresponding operation. If MCU needs to upload data to a PC, just needs to reverse the process. The CAN controller hardware schematic diagram is shown in Fig. (3).

\subsection{Hardware Design of Motor Driver Board}

Stepper motor is an electrical change pulse to the angular displacement of the actuator, that is, when the stepper driver receives a pulse signal, it will drive a stepper motor to set the direction of rotation of a fixed angle (i.e. step angle).Stepper motor controls the amount of angular displacement by controlling the pulse number, so it can achieve accurate positioning. Meanwhile, by controlling the speed and acceleration of the motor rotation, Stepper motor can achieve the purpose of speed control [7].

The system is designed to be able to driver 4 stepper motor by one motor board, and each stepper motor is driven by L297 + L298 module.

The signal that the stepper motor controller send is not directly transfer into L297, but through the chip TLP521.TLP521 is a photoelectric coupling device, which can be used for signal transmission between the transmission circuit, control circuit between the signal, so that the input and output end completely isolated, this can increase safety and reduce the circuit interference of the system. TLP521 unit is shown in Fig. (4). 


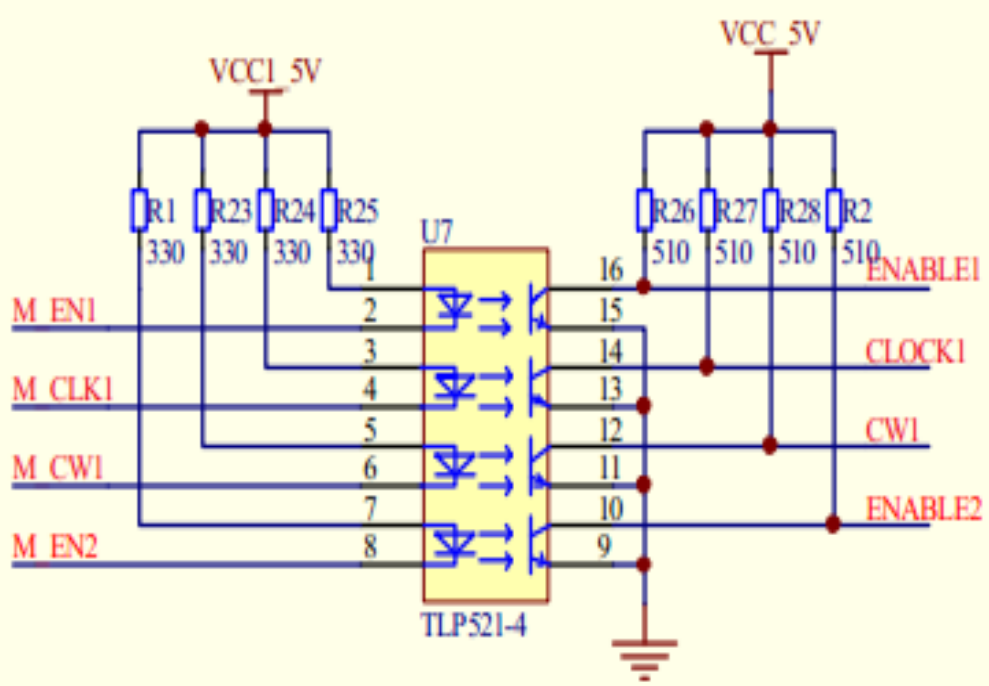

Fig. (4). Motor driver circuit.

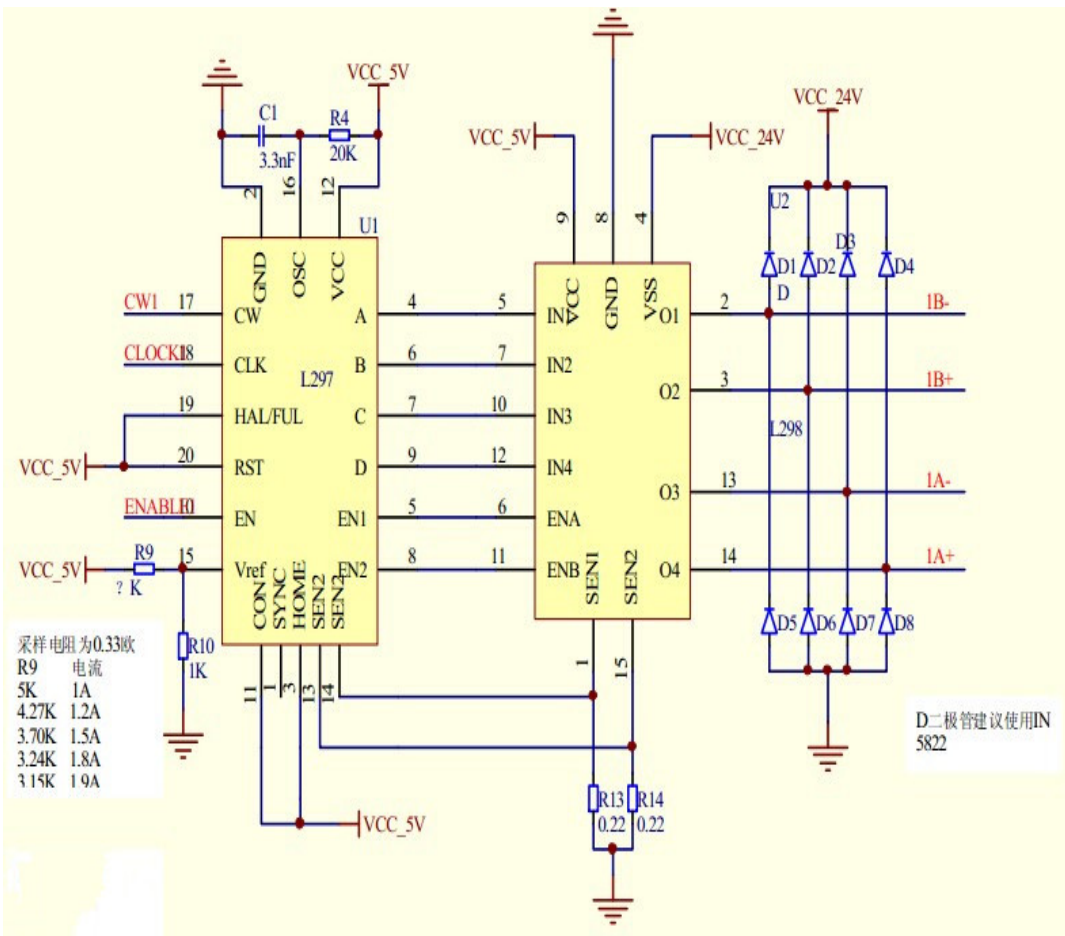

Fig. (5). Stepper motor drive circuits.

L297 internal integrated chopping constant current driving circuit, which can improve the power efficiency, effectively restrain the resonance and improve the speed characteristics, so the pressure head can achieve accurate displacement. By adding the RC circuit on L297 OSC pin and adding comparing voltage on the VREF, the system can control the chopper frequency, then control the stepping motor phase current size.
As shown in Fig. (5), the control chip L297 is mainly used to receive the pulse emitted by the ATmega64, and then drive the L298 chip. The L298 chip is a high voltage, high current dual full bridge driver. It was designed for receiving the standard TTL logic level signal and driving inductance load. It has two inhibitory inputs to the device from the impacts of input signal. It can also be seen that each phase coil of stepper motor ends is connected a reverse diode, it can 


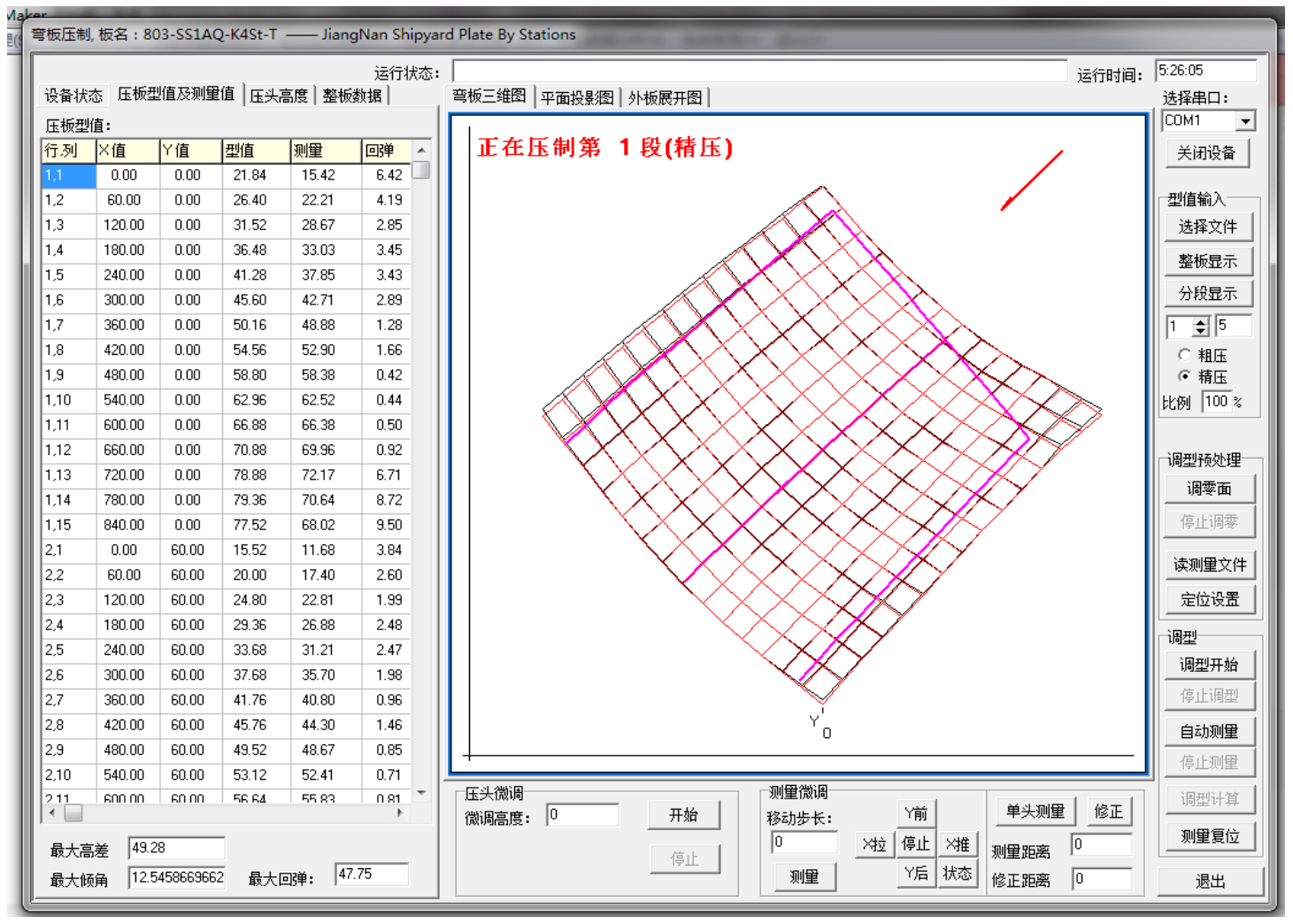

Fig. (6). Control software interface.

relief induced electromotive of the motor coil, and then avoid the motor from overheating [8].

\section{DESIGN OF CONTROL SOFTWARE}

\subsection{Design of PC Control Software}

PC control software is written by using $\mathrm{VC}++6.0$ tools. $\mathrm{VC}++6.0$ visualization, which has friendly interface and powerful functions. The system mainly used the structure of multi view single document [9]. In the $\mathrm{VC}++$ environment, by using Windows API function to the research and development of the pressing machine control interface based on CAN bus, the control software can import the shape adjusting data which generated by $\mathrm{CAD} / \mathrm{CAM}$ software, and then send the control commands via the CAN bus to the lower computer, finally the sheet metal can be formed. Control software interface is as shown in Fig. (6).

In the software interface, monitoring window is divided into four parts. The first part is the equipment status area, which both shows the progressive display plate type value in the form of table and shows that the maximum difference, the maximum angle, the maximum rebound. The second part is the graphics display area, which shows the plate bending $3 \mathrm{D}$ graph, plane projection drawing, the outer plate projection. The third part is setting and controlling area, which can adjust the single head according to the fine-tuning of stamping and metal material position feedback. The fourth part is the software configuration area, which used for serial port configuration, file import of software and so on.

\subsection{Software Design of the Lower Computer}

The lower computer control software used AVR Studio 4 development environment, and used the $\mathrm{C}$ language as the programming language. The lower computer receives CAN data sent by PC, then parse the frame of ID, data content, and get the control pressure head number, direction, number of steps which need to be controlled, and then generates the corresponding pulse output to the corresponding stepper motor drive circuit, then driving head achieve punching.

Take the motor rotation instruction as an example, the frame ID of it is $0 \times 539$. According to the provisions of CAN bus, the effective number of bytes of each frame is 8 . Specific data meaning are as defined in Table $\mathbf{1 .}$

Table 1. Motor control CAN instruction packet definition.

\begin{tabular}{|c|c|c|}
\hline Points & Place of Text & Type Styles \\
\hline \hline 7 & Motor horizontal coordinates & $0 \sim 14$ \\
\hline 6 & Motor longitudinal axis coordinates & $0 \sim 14$ \\
\hline 5 & Motor rotation direction & $\begin{array}{c}0: \text { Forward; } \\
\text { 1: Backward }\end{array}$ \\
\hline 4 & Motor step number high byte & \\
\hline 3 & Motor step number low byte & \\
\hline 2 & Reserve & \\
\hline 1 & Reserve & \\
\hline 0 & Reserve & \\
\hline
\end{tabular}




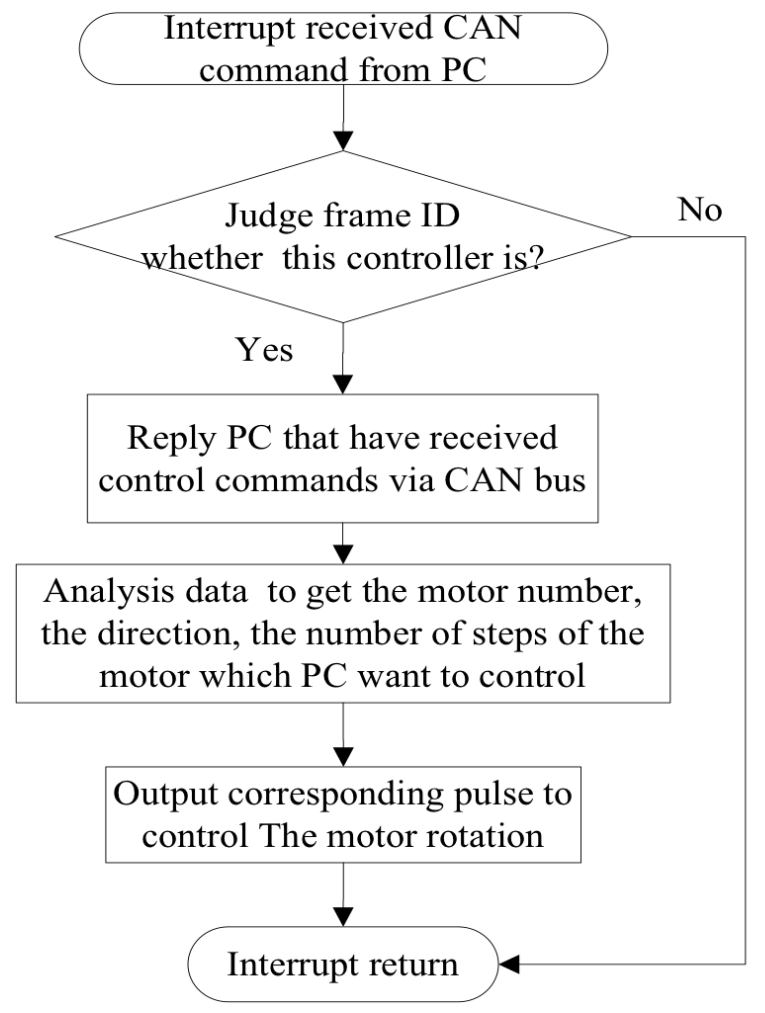

Fig. (7) The working process of the lower computer.

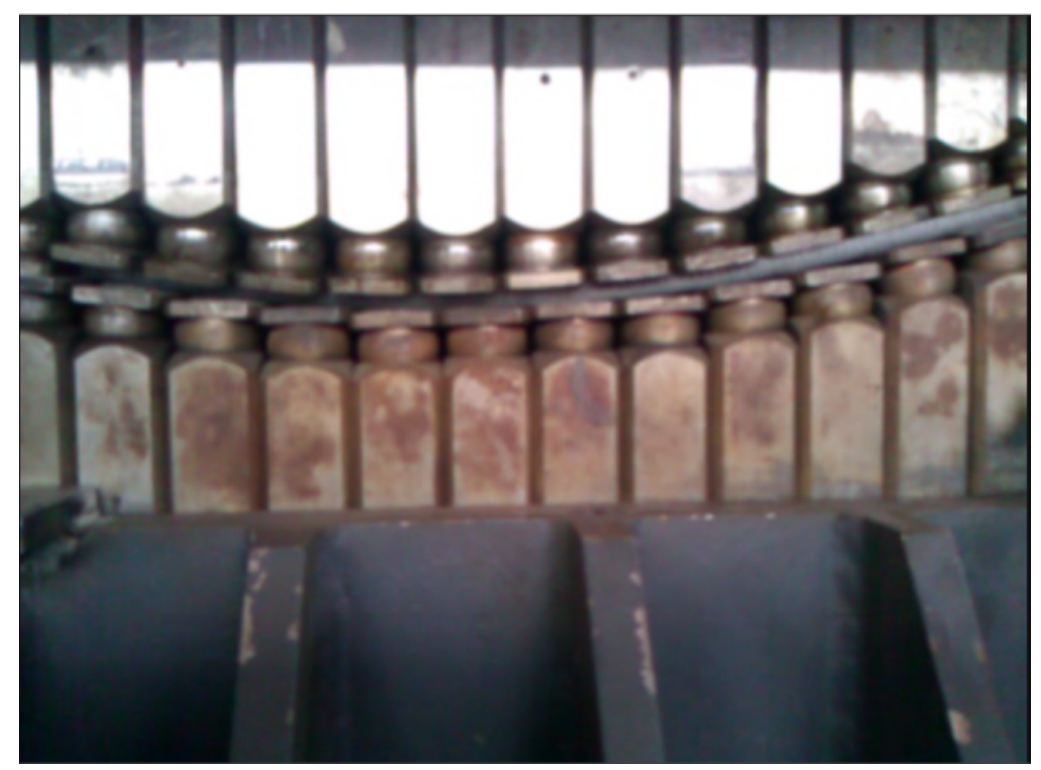

Fig. (8) Platen machine control system.

Control flow chart is shown in Fig. (7).

After completion, the up mold is removed, using the measuring device measures the pressure at the head of each sheet forming a shape, and compare the shape with the contrast theory, calculate the shape error, then prepare for the next tune-shaped.

\section{EXPERIMENTAL RESULT}

After the system is assembled and debugged, the hyperbolic degree platen machine was run and an experiment to processing a ship hull plate was made. As shown in Fig. (8) and Fig. (9), the effect of stepping motor control system based on CAN bus technology is good, high control 


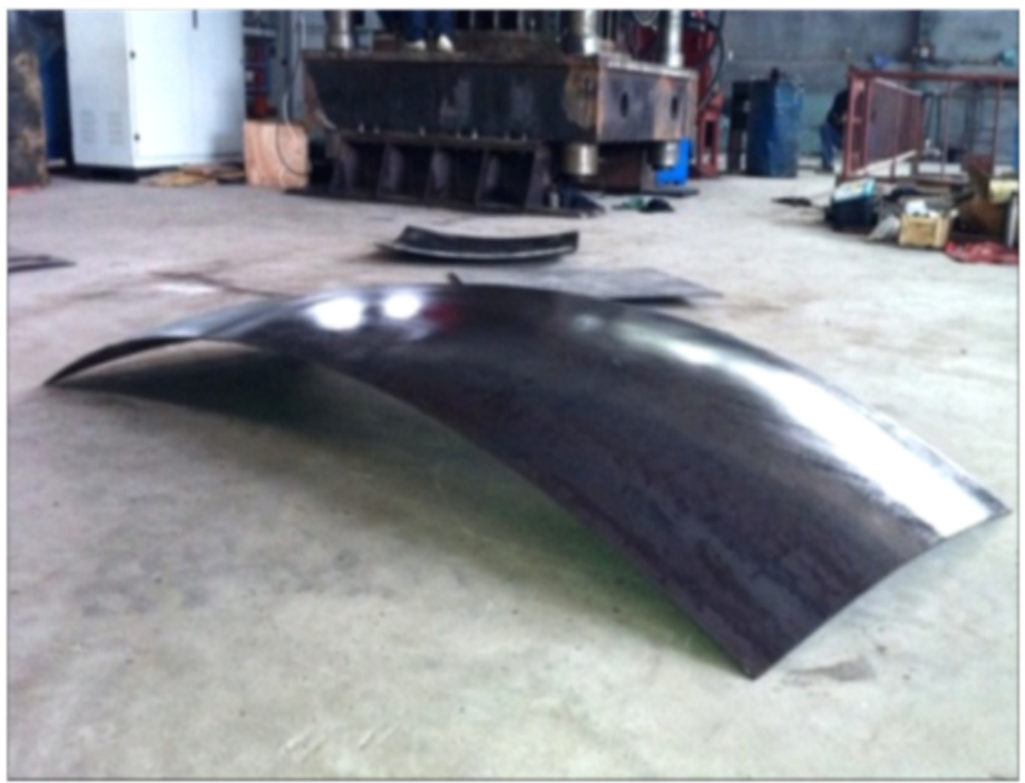

Fig. (9) Platen machine control system test results.

accuracy, thus the sheet surface is smooth, wrinkle-free phenomenon. Due to the use of height adjustable square heads, there was no indentation on the sail plate which shaped by stamping.

\section{CONCLUSION}

The design of hyperbolic ship hull plate press machine control system based on CAN bus, used a hardware system which consisted by a master MCU ATmega64, CAN controller and CAN transceiver PCA82C250T SJA1000T CAN controller and L297 + L298 stepper motor driver. It also composed a PC control monitoring software based on $\mathrm{VC}++$ 6.0. The system achieves a set of hyperbolic degree hull plate processing platen machine stepper motor control system which running well, stable and reliable. The experiment proved that the system can effectively improve the quality of sheet metal forming, increase productivity, improve working conditions, mechanization, automation, and digital production for the hyperbolic shape of the hull plate provides a new way and programs.

\section{CONFLICT OF INTEREST}

The authors confirm that this article content has no conflict of interest.

\section{ACKNOWLEDGEMENT}

This work is supported by the Education Department of Hubei Province Science and Technology Research Projects,
China (No.Q20141501), the Research Fund of NSFC, China (No.51379167).

\section{REFERENCES}

[1] J. F. Hua, Y. Hu, C.F. Wang, and Y. Q. Zhou, "No-pair dins forming technology for complex curved surface of ship hull plate", Ship Engineering, vol. 33, pp. 63-66, 2011.

[2] Z. H. Chen, M. Z. Li, "Numerical simulation application in multipoint forming". International Conference on Mechatronics and Automation,CMA, pp. 2250-2254, 2009.

[3] H. H. Wei, G. J. Chen, Y. B. Zhang, and L. Li, "Design of stepper motor control system based on CAN bus". Automation and Instrumentation, pp. 29-32, 2009.

[4] S. H. Hong, W.H. Kim, "Band width allocation scheme in CAN protocol". IEEE Proceedings on Control Theory and Applications, vol. 147, pp. 37-44, 2000.

[5] D. Zhou, M. G. He, and F. X. Yang, "Design of Stepper motor controlled module based on CAN and AVR". Measurement and control technology, vol. 28, pp. 71-74, 2009.

[6] D. Zhou, "Design and implementation of automatic biochemical analyzer control system". Beijing University of Posts and Telecommunications, 2009.

[7] M. Xia, X. T. Yu, and X. Y. Yuan, "The design of locomotive wheel tread detection system based on AVR microcontroller. Journal of WUT", Information \& Management Engineering, vol. 32, pp. 78-80, 2010.

[8] T.S. Weerakoon and L. Samaranayake, "Development of a Novel Drive Topology for a Five Phase Stepper Motor". The third international Conference on Industrial and information systems pp. $1-6,2008$.

[9] Y. B. Zhang and J. X. Wang, "Development of the measuring and controlling system for temperature data based on VC6.0". Machinery and Electronics, pp. 46-48, 2006.

(C) Xiong et al.; Licensee Bentham Open.

This is an open access article licensed under the terms of the Creative Commons Attribution Non-Commercial License (http://creativecommons.org/licenses/by-nc/3.0/) which permits unrestricted, non-commercial use, distribution and reproduction in any medium, provided the work is properly cited. 